\title{
La gastronomía como atractivo turístico primario en el centro histórico de Quito
}

\section{Gastronomy as a primary tourist attraction in the historic center of Quito}

Juan F. Romero-Corral

Universidad Iberoamericana del Ecuador, Ecuador

Autor para correspondencia: jromero@unibe.edu.ec / 0984878194

Fecha de recepción: 28 de agosto de 2018 Fecha de aceptación: 15 noviembre del 2018

Resumen: El presente trabajo aborda la gastronomía del centro histórico de la ciudad de Quito como un atractivo turístico primario, una de las principales motivaciones de confluencia tanto de turistas extranjeros como nacionales. El centro histórico de la ciudad de Quito es uno de los atractivos más visitados en Ecuador, ya que es considerado como el más grande y conservado de la región, además también su culinaria constituye una de las principales motivaciones para su visitación. Este trabajo da a conocer las principales características que han permitido que se desarrolle un turismo gastronómico en el centro de esta ciudad, tendencia que en la actualidad incluye elementos culturales, históricos, de saberes y sabores tradicionales que han puesto en valor la diversidad de productos, preparaciones y oferta gastronómica que son parte de la dinámica alimenticia de su cotidianidad. La creación de una ruta gastronómica demuestra la amplia oferta que permite consolidar a la cocina quiteña en este Centro Histórico como un atractivo turístico primario. Para ello, se realizó una encuesta a 100 personas, con preguntas abiertas, cerradas y de opinión utilizando el Diseño Explicativo Secuencial (DEXPLIS). Se concluye que el turismo gastronómico es una herramienta para conocer el patrimonio material e inmaterial de una localidad.

Palabras clave: turismo gastronómico; patrimonio cultural; ruta gastronómica; Quito

Abstract: This work analyzes the gastronomy of Quito's downtown as a primary tourist attraction which is one of the main motivations of the confluence of foreign and national tourists. This historic downtown is one of the most visited attractions in Ecuador because its size and its best preservation. Furthermore, its culinary is one of the main motivations to visit it. This work shows the main characteristics which have allowed the development of the Gastronomic Tourism in Quito's downtown. This tendency includes a variety of elements of culture, history, knowledge and traditional tastes which are worth to see, such as: the diversity of products, preparations, and the gastronomic offer which are part of its daily food dynamics. The creation of a gastronomic route shows the wide offer that allows consolidating the cookery of Quito's downtown as a primary tourist attraction. Besides, it was applied a survey to a sample of 100 people in which they were asked with open and closed questions. Moreover, it contained opinion questions by using the Diseño Explicativo Secuencial (DEXPLIS) (Sequential Explanatory Design) in order to get results. This research concludes that the Gastronomic Tourism is a tool to know the material and immaterial heritage.

Key words: gastronomic tourism; cultural heritage; gastronomic route; Quito 


\section{Introducción}

En la actualidad, el turismo gastronómico es una herramienta que ha permitido a algunas regiones brindar lo mejor de sí al momento de atraer a los turistas, tomando en cuenta que sus motivaciones para trasladarse a un destino giran en torno a la idea primordial de degustar la variada oferta gastronómica de tal sitio. Dentro de este contexto, la principal motivación de visitación ya no parte solo de conocer los atractivos turísticos naturales o arquitectónicos de una ciudad o región, sino más bien de internarse en un mundo de costumbres y tradiciones que solo la culinaria regional puede brindar, basada en la sostenibilidad y el manejo responsable de recursos, protección del ambiente y la salvaguarda de los valores patrimoniales y culturales (OMT, 2017).

El objetivo general de la presente investigación fue el de determinar si la gastronomía del centro histórico de la ciudad de Quito puede o no ser considerada un atractivo turístico primario. Para ello, se analizaron: los factores que han permitido el incremento del número de visitantes a este centro histórico debido al turismo gastronómico y se propuso también la consolidación de una ruta gastronómica que considere como eje central los productos gastronómicos más importantes del sector.

\section{El turismo gastronómico}

El centro de la ciudad de Quito presenta las condiciones propicias para ser promocionado como un atractivo turístico, sobre todo por los recursos gastronómicos debido a que la cocina que se oferta es reconocida por los visitantes; según Ascanio (2009) un elemento turístico importante es la gastronomía local orientada a un segmento de mercado definido. Entiéndase el turismo culinario no como la necesidad de comer, sino con varios elementos enlazados: el ambiente, la infraestructura del espacio de servicio, la atención, la uniformidad, los colores y olores que transmiten los saberes y sabores a quien los visita.

Según la Organización Mundial del Turismo, el turismo se define como:

Fenómeno social, cultural y económico relacionado con el movimiento de las personas a lugares que se encuentran fuera de su lugar de residencia habitual por motivos personales o de negocios/profesionales. Estas personas se denominan visitantes (que pueden ser turistas o excursionistas; residentes o no residentes) y el turismo tiene que ver con sus actividades, de las cuales algunas implican un gasto turístico (OMT, 2017).

Por otra parte la actividad gastronómica se encuentra estrechamente entrelazada con la idea de que:

Los alimentos son todas las sustancias que, sometidas al estómago, pueden animalizarse por la digestión y reponer las pérdidas que el cuerpo humano experimenta en el trascurso de la vida“(Brillat, 2010, pág. 72). 
Por tanto, el turismo gastronómico es un tipo de turismo en el que la gastronomía del país visitado es el hecho fundamental que guía la visita. Este tipo de turismo, se presenta hoy como una opción alternativa (www.vix.com).

Cuando se habla del centro histórico de la ciudad de Quito, el turista lo relaciona inmediatamente con su arquitectura colonial y barroca, pero su cultura culinaria ha estado siempre latente, es por eso que muchas de sus conocidas calles tienen algo más que ofrecer: justamente, su gastronomía. El presente estudio busca conocer la vasta oferta gastronómica que se produce en este sector de la ciudad y determinar el grado de influencia en la decisión del turista para considerar a este como un atractivo turístico primario siendo una las principales motivaciones para la llegada de los visitantes, tanto nacionales como extranjeros.

La culinaria de la región antes de la llegada de los españoles fue una dieta basada básicamente en granos, vegetales, hortalizas, como por ejemplo el maíz y los conocidos RTAs (Raíces y Tubérculos Andinos) (Bonete et al., 2016) y fauna endémica como la vicuña, la alpaca, el cuy y la hualpa productos estos últimos mayormente consumidos por los incas en sus ceremonias. "La Villa de Quito, fundada de manera definitiva el 6 de diciembre de 1534, fue el escenario en el que se efectuaron las primeras experiencias de adaptación de especies domésticas traídas de Europa por el sacerdote franciscano Fray Jodoco Ricke” (Trujillo, 2017, pág. 175).

Los principales productos con la llegada de España a América fueron la caña de azúcar, el trigo, el ganado vacuno y sus derivados, los mismos que con el pasar del tiempo configurarían la cocina quiteña desde el tiempo de la Colonia hasta nuestro tiempo (Pazos-Barrera, 2010). Quito a inicios del siglo XVII fue considerada en la región interandina ecuatoriana como la primera ciudad productora de derivados de leche, específicamente en las queserías.

Según Pazos-Barrera (2010), la gran mayoría de los productos elaborados en la ciudad de Quito son el resultado de una fusión de ingredientes. En lo que hoy conocemos como la Iglesia de San Francisco se encontraba el tiánguez más importante, junto con el de la ciudad del Cuzco, posiblemente los más grandes y abastecidos de Sudamérica. Debido al intercambio comercial con poblaciones de la Sierra, de la Costa y el Oriente, además el intercambio de utensilios y conocimientos tanto de técnicas, procedimientos y métodos de conservación y elaboración, los mismos que fueron enriqueciendo la herencia culinaria que se formó en la ciudad.

Con las primeras elaboraciones culinarias realizadas en Quito y con algunas realizadas en otras regiones de Ecuador se empiezan a crear los primeros emprendimientos culinarios, muchos de ellos adaptaciones en zaguanes para el proletariado y otros construidos específicamente para la elaboración de alimentos y bebidas, lo que va dando lugar a bulevares gastronómicos en la ciudad de origen artesanal, debido a la necesidad de contar con establecimientos para atender a la nueva clase burguesa que se erige en la ciudad de Quito, gracias al desarrollo de la cocina local en los siglos XVII y XVIII.

Benítez (1995) menciona que en el gobierno de Gabriel García Moreno, se expresó

"partidario de que este país magnífico llegue a ser civilizado y rico bajo la bandera de Francia; aspiro a que el pueblo del Ecuador sea tan feliz que se una él mismo a esa grande y 
generosa nación" (pág. 197). A pesar de la fidelidad de la aristocracia quiteña al Rey Felipe de España, se incorporó a la gastronomía local productos traídos de Francia, Además trajeron consigo sus técnicas de elaboración y conservación, conocimientos desarrollados tanto por las distintas ordenes católicas como por profesionales y cronistas gastronómicos de la época siendo este antecedente parte de la etapa republicana de la cocina quiteña (Ministerio de Cultura y Patrimonio, 2016).

Esto se reflejaba en escritos como el siguiente: "En esto vinieron a avisarnos que el almuerzo nos esperaba. Fue excelente. Preparado por un buen cocinero francés y servido por cocineros franceses, nada nos dejó que desear. Al final saboreamos un magnífico moca" (Avendaño, 1857, citado por Pazos Barrera, 2010).

La ciudad de Quito no posee vastos registros o evidencias de recetas vernáculas, la mayoría de información encontrada son recetarios con productos y preparaciones coloniales, los mismos que se encontraron en monasterios y en bibliotecas familiares ubicadas en el centro histórico, se podría suponer que estas recetas han permitido la construcción del patrimonio culinario de la ciudad de Quito, siendo parte del imaginario social en lo que se refiere a ritos y tradiciones, propios de su cotidianidad. Según Unigarro (2010) estos conocimientos sufren constantes mutaciones y transformaciones de generación en generación, transformándose en catalizadores de desarrollo social, cultural y económico, convirtiéndose en parte del patrimonio inmaterial de una localidad.

Por esta razón es importante mencionar que al no existir muchos documentos de dominio público, estos insumos se encuentran registrados desde sus inicios en libros o cuadernillos culinarios, pero era lo más común que estos conocimientos fueran replicados de generación en generación de una forma oral y prestándose a la buena o mala interpretación de quién los preparaba.

Debido a esto, tanto para profesionales del turismo como de la gastronomía de esta urbe es de imperiosa necesidad investigar, discernir y poner en valor a estas preparaciones, debido a que estas recogen la parte identitaria de la culinaria local. Estas investigaciones podrían permitir conectar a la academia, al Estado (Ministerio del Turismo del Ecuador), al gobierno municipal (Quito Turismo) y a la industria turística, hotelera y restaurantera, con la finalidad de crear políticas que impulsen un aumento de la visitación sostenible a los lugares turísticos del centro histórico de la ciudad como: iglesias, museos, teatros, callejuelas y además el disfrute de las actividades complementarias como el consumo de la gastronomía local potenciando la economía de los lugares ya existentes, o permitiendo la creación de nuevos emprendimientos afianzando el aporte culinario moderno.

"El que visita por primera vez una ciudad lleva en sí un tesoro de ignorancia junto a otro no menos grande, de evidencia. En verdad ignora todo de la nueva ciudad, por muy bien informado que esté. Hay un alma dentro de cada agrupación humana, un ambiente una "manera", que no se revelan sino después de una larga convivencia. Esta alma es todo; por ella se explica todo lo que la ciudad es y lo que ella encierra" (Subercaseaux, 1948, págs. 146- 147) 
Una de las principales estrategias que utilizan las ciudades y comunidades para fomentar el turismo gastronómico son los festivales culinarios, la creación de rutas en los cuales se da a conocer los restaurantes donde se elaboran las principales preparaciones de la región, más aun cuando son preparadas por un chef reconocido de la localidad explicando todo el proceso desde la recolección y/o caza de los productos y finalizando con los sutiles toques al momento de montar un plato de autor (Hall \& Mitchell, 2005); el turismo gastronómico establece una relación entre un determinado conglomerado de personas, produciendo un fenómeno cultural, patrimonial, económico y ambiental.

\section{Turismo nacional y extranjero en la ciudad de Quito}

El Centro Histórico es el destino turístico más visitado en la capital. Luego están los parques y plazas, el teleférico, el Panecillo, la Mitad del Mundo, la Mariscal, según estadísticas que maneja la entidad municipal Quito Turismo (2013, pág. 11).

\section{Métodos}

En la actualidad, la gastronomía se ha convertido en una importante estrategia para atraer turistas a las localidades, siendo el turismo gastronómico una de las principales alternativas en las preferencias de los consumidores. Según Stewart et al. (2008), la principal motivación de los turistas es la experimentación de nuevos saberes y sabores al recorrer las rutas gastronómicas diseñadas para conocer la cultura culinaria de una región.

El presente artículo describe resultados de una investigación realizada en el centro histórico de la ciudad de Quito, desde julio hasta agosto de 2014, en torno al turismo gastronómico como un atractivo turístico primario, debido a que este sector patrimonial de la ciudad es el que registra mayor número de visitantes tanto nacionales como extranjeros. Se realizaron encuestas a turistas nacionales y extranjeros, de acuerdo a lo descrito en los siguientes párrafos.

\section{Población}

Aproximadamente 618 mil extranjeros, principalmente de USA, Colombia, Perú, Alemania, Reino Unido y 1'000.000 de nacionales visitan la capital cada año (Quito Turismo, 2016, pág. 25). A eso se suman entre 500 mil y 600 mil personas que viviendo en la capital hacen turismo interno.

Según Quito Turismo (2013), se atribuye la presencia turística a la posición cercana de la ciudad a países fronterizos, así como su estabilidad económica en referencia a Colombia o Perú. Para este año se aspira a aumentar su flujo entre el $4 \%$ y $5 \%$, mientras que el año anterior fue de entre el $2 \%$ y $3 \%$.

\section{Muestra y técnica aplicada}

Entre julio a agosto de 2014 (época de mayor visitación anual de turistas a Quito), se aplicaron en algunos sectores del centro histórico (Calles: Rocafuerte, Guayaquil, Benalcázar, 
Espejo; Plazas: San Francisco, Santo Domingo, de la Independencia) 100 encuestas a 25 turistas adultos extranjeros (norteamericanos y europeos) y 75 a turistas adultos nacionales.

La técnica aplicada fue la encuesta mixta, preguntas cerradas y preguntas de opinión, a través del Diseño Explicativo Secuencial (Dexplis), mismo que según Hernández et al. (2014) se caracteriza porque "en la primera etapa se recaban y analizan datos cuantitativos, seguida de otra donde se recogen y evalúan datos cualitativos. La mezcla mixta ocurre cuando los resultados cuantitativos iniciales informan a la recolección de los datos cualitativos” (Pág. 544).

Este método se aplicó en las encuestas a turistas, dando énfasis a interrogantes sobre turismo gastronómico y la creación de una ruta gastronómica a partir de los principales productos, preparaciones y restaurantes que crean motivación para su visitación. Los instrumentos a utilizarse fueron:

\section{Lista de cotejo}

Este instrumento permitió registrar la presencia o ausencia de una determinada conducta, secuencia de una acción o rasgo. Es dicotómica, es decir acepta únicamente dos alternativas: aplica, no aplica; procede, no procede o la más común dentro de una encuesta las alternativas Sí o No.

\section{Entrevista semi estructurada}

Según Hernández et al. (2014), las entrevistas semi-estructuradas, son una guía de preguntas y asuntos en la cual el entrevistador cuenta con la posibilidad de elaborar preguntas adicionales para obtener mayor información sobre temas deseados. Para esta investigación se desarrollaron preguntas buscando que los encuestados den su opinión y apliquen su conocimiento sobre lo que entienden por turismo gastronómico, principales platos, restaurantes y la necesidad de una ruta gastronómica.

\section{Resultados y Discusión}

El análisis de las respuestas dadas por las 100 personas abordadas a continuación:

\section{Pregunta 1:}

¿Conoce usted qué es una ruta gastronómica?

\begin{tabular}{lll}
\hline & SI & NO \\
\hline femenino de $20-40$ años & 2 & 26 \\
masculino de $20-40$ años & 5 & 19 \\
femenino de $41-65$ años & 5 & 15 \\
masculino de $41-65$ años & 4 & 24 \\
SUMATORIA & $\mathbf{1 6}$ & $\mathbf{8 4}$ \\
\hline
\end{tabular}

El 84\% de los encuestados opinan que no tienen conocimiento de lo que es una ruta gastronómica por la falta de información o difusión; la encuesta determina que resulta importante 
generar una ruta gastronómica en torno a la gastronomía típica quiteña en el centro histórico de Quito y darla a conocer ampliamente.

\section{Pregunta 2:} conoce?

¿Cuáles son los restaurantes más representativos del centro histórico de Quito que usted

Las respuestas fueron:

- Las Guatitas de La Colmena.

- Heladería San Agustín.

- Hasta la Vuelta Señor.

- Mea Culpa.

- La Casa de la Peña

- El Criollo.

- Taita Pendejadas.

- Las Empanas de Morocho de la Olmedo.

- Vista Hermosa,

- El Escondite de Cantuña.

Los encuestados señalaron estos lugares como más representativos; estos expenden comida típica quiteña. También los encuestados mencionaron otros sitios que expenden comida no tradicional de Quito, que resultan no relevantes en esta investigación.

\section{Pregunta 3:}

De los siguientes: ¿Cuáles platos o preparaciones quiteñas conoce?

- $\quad$ Rosero Quiteño.

- Locro de papas.

- Colaciones.

- Higos con queso.

- Seco de Chivo.

- Canelazo.

- Mistelas.

- $\quad$ Locro de Papas.

El $90 \%$ de los encuestados manifestaron que conocían todos los platos quiteños nombrados. El 10\% mencionó conocer máximo 6 de ellos.

\section{Pregunta 4:}

¿Le agradaría conocer cuáles son los mejores restaurantes que expenden comida quiteña típica dentro del Centro histórico de Quito?

El 97\% de los encuestados afirma que les gustaría conocer los mejores sitios de comida tradicional en el centro histórico de Quito por numerosas razones, principalmente degustar sus platos. 
El 3\% mencionó que no consumen la gastronomía del centro histórico, debido a que les parece muy repetitiva la oferta.

\section{Pregunta 5:}

Una Ruta Gastronómica indica los lugares que expenden platos típicos y su ubicación dentro del centro histórico. ¿Considera usted que la presencia de esta ruta incentivaría el turismo gastronómico en el sector?

\begin{tabular}{lll}
\hline & SI & NO \\
\hline femenino de 20 - 40 años & 1 & 27 \\
masculino de 20 - 40 años & 2 & 24 \\
femenino de 41 - 65 años & 1 & 22 \\
masculino de 41 - 65 años & 2 & 21 \\
SUMATORIA & $\mathbf{6}$ & $\mathbf{9 4}$ \\
\hline
\end{tabular}

El 6\% de los encuestados afirma que la creación de una ruta gastronómica no sería un mecanismo para incentivar el turismo en el sector. El 94\% por su parte manifestaron que la existencia de una ruta gastronómica formal es la mejor manera de incentivar la visitación tanto de turistas nacionales como de extranjeros.

\section{Pregunta 6:}

¿Le gustaría que exista un manual de rescate de productos y preparaciones que ya no se utilizan o se preparan muy poco en la actualidad en Quito?

\begin{tabular}{llc}
\hline & SI & NO \\
\hline femenino de $20-40$ años & 27 & 1 \\
masculino de $20-40$ años & 29 & 1 \\
femenino de 41 - 65 años & 22 & 1 \\
masculino de 41 - 65 años & 19 & 0 \\
SUMATORIA & $\mathbf{9 7}$ & $\mathbf{3}$ \\
\hline
\end{tabular}

E1 97\% de los encuestados afirmó que sí le gustaría que exista un manual de productos y preparaciones de la comida tradicional del centro histórico de Quito.

El 3\% restante manifestó que no necesita conocer esta información ya que no consume ningún tipo de comida típica en el centro de la ciudad.

\section{Pregunta 7:} de Quito?

¿Por qué cree que es necesario implementar una ruta gastronómica en el centro histórico

La mayoría de los encuestados manifestaron que es muy importante implementar una ruta gastronómica en este sector de la ciudad, debido a que hay muy poca información sobre los productos culinarios típicos que se expenden en este sector, a los encuestados les parece un importante aporte para incrementar la cantidad de visitantes.

\section{Pregunta 8:}


¿Cómo desearía obtener información acerca de una Ruta Gastronómica del Centro Histórico de Quito?

Por medio de trípticos: $10 \%$ de los encuestados.

Por medio del e-mail: $15 \%$.

Por medio de Islas Informativas dentro del centro histórico: $20 \%$.

En los propios establecimientos del centro histórico de Quito: 55\%.

\section{Conclusión}

En el lugar de estudio se puede apreciar la profunda relación que existe entre su gastronomía y sus visitantes, siendo la primera una de las principales actividades económicas que se desarrollan allí de manera cotidiana.

La conformación de un documento (impreso o digital) que detalle formalmente una Ruta Gastronómica en el centro histórico de Quito es una herramienta que permitirá mostrar y conocer de manera sistemática los lugares más importantes en los que se desarrolla la gastronomía típica del sector, siendo un elemento de gran ayuda para la orientación de sus visitantes, permitiendo un turismos gastronómico más ordenado y sostenible.

La vasta historia en la que se ha desarrollado la culinaria de la ciudad de Quito permite que la gastronomía local sea un factor de alta motivación física y cultural para su visitación, debido a que en el recorrido a cada uno de los lugares de expendio de alimentos existe una historia sobre sus principales productos, procedimientos y técnicas empleadas, la misma que hace incuestionable su carácter patrimonial.

Una de las obligaciones de la academia y la industria es la puesta en valor de los principales productos y preparaciones con la finalidad de fomentar en los estudiante y emprendedores una identidad cultural gastronómica, siendo los principales promotores para dar a conocer la culinaria de la ciudad, tanto a turistas nacionales como extranjeros, brindando servicio de calidad tanto en aspectos de inocuidad alimentaria y atención al cliente.

En este sentido, retomando lo propuesto por Oliveira (2010) para sitios similares, la gastronomía en este sector (centro histórico de Quito) estaría ligada más con la curiosidad y el "excursionismo", siendo éste un sitio de visitación de elementos culturales e históricos, pero en el que la degustación de preparaciones culinarias típicas presentaría las condiciones ideales para convertirse en un atractivo turístico primario.

\section{Bibliografía}

Ascanio, A. (2009). Rutas gastronómicas chilenas: una aproximación al tema. Pasos, 7(2), 321-325.

Benítez, L. (1995). Ecuador: drama y paradoja. Quito: Ensayo.

Bonete, M., Urquizo, C., Guevara, R., \& Yánez, P. (2016). Estudio de cuatro tubérculos y raíces tuberosas no tradicionales de la sierra centro de Ecuador y su potencial de uso en platos de autor. Qualitas, 12, 37-67. 
Brillat, S. (2010). Fisiología del gusto. Madrid: Maxtor.

Hall, C., \& Mitchell, R. (2005). Gastronomic tourism: comparing food and wine tourism experiences. Routledge.

Hernández, R., Sampieri, C., \& Baptista, P. (2014). Metodología de la Investigación. México D.F: McGraw- Hill.

Ministerio de Cultura y Patrimonio. (24 de Junio de 2016). La cocina republicana y la revolución gastronómica moderna. Obtenido de: http://patrimonioalimentario.culturaypatrimonio.gob.ec/wiki/index.php/La_cocina_republican a_y_la_revoluci\%C3\%B3n_gastron\%C3\%B3mica_moderna

Oliveira, S. (2010). La gastronomía como atractivo turístico primario de un destino: el Turismo Gastronómico en Mealhada - Portugal. Recuperado el 06 de septiembre de 2017 de: http://www.scielo.org.ar/scielo.php?script=sci_arttext\&pid=S1851$17322011000300012 \& \operatorname{lng}=$ es\&nrm=iso.

OMT: Organización Mundial del Turismo. (17 de mayo de 2017). Segundo informe de la OMT sobre turismo gastronómico: sostenibilidad y gastronomía. Recuperado el 12 de septiembre de 2017, de: http://media.unwto.org/es/press-release/2017-05-25/segundo-informe-de-la-omt-sobreturismo-gastronomico

OMT: Organización Mundial del Turismo. (Sin año). Entender el turismo: Glosario Básico. Recuperado el 06 de septiembre de 2017, de: http://media.unwto.org/es/content/entender-elturismo-glosario-basico

Pazos-Barrera, J. (2010). El Sabor de la Memoria. Quito: Fonsal.

Quito Turismo. (2013). Quito en cifras. Quito: Municipio de Quito.

Quito Turismo. (2016). Quito en cifras. Obtenido de: http://inversiones.quito.com.ec/wpcontent/uploads/2016/12/quito-en-cifras_DIC2016.pdf

Stewart, J., Bramble, L., \& Ziraldo, D. (2008). Key challenges in wine and culinary tourism with practical recommendations. International Journal of Contemporary Hospitality Management, 20(3), 302-312.

Subercaseaux, B. (1948). Una locageografía. Santiago de Chile: Ercilla.

Trujillo, J. (2017). La Fanesca, Antropología de la Culinaria Ritual Ecuatoriana. Quito: Foncultura.

Unigarro, C. (2010). Patrimonio Cultural Alimentario. Quito: La Tierra.

www.vix.com/es/imj/mundo/2008/11/20/turismo-gastronomico. Recuperado el 06 de septiembre de 2017. 